\title{
A 5 year trend analysis of malaria prevalence with in the catchment areas of Felegehiwot referral Hospital, Bahir Dar city, northwest-Ethiopia: a retrospective study
}

\author{
Mulat Yimer ${ }^{1 *}$, Tadesse Hailu', Wondemagegn Mulu', Bayeh Abera and Workneh Ayalew²
}

\begin{abstract}
Background: Malaria is one of the killer diseases in Ethiopia and it is still the first leading cause of death in health facilities. However, there is no information yet regarding the trends of malaria prevalence at health institution and particularly at Felegehiwot referral Hospital. Hence, knowing the trends of malaria prevalence at each health facilities is essential to design appropriate interventions. Therefore, the present study addressed the above gaps.

Results: Overall, 14,750 blood films were diagnosed for malaria. Of these, 740 (5\%) were confirmed with microscope. Plasmodium falciparum and Plasmodium vivax accounted for 397 (53\%) and 331 (45\%), respectively. Age groups $>20$ year $(p<0.02)$ and males $(p<0.025)$ were significantly affected.

Conclusions: In conclusion, P. falciparum was predominant as compared to $P$. vivax. Hence, it needs close monitoring and intervention measures for control activities.
\end{abstract}

Keywords: Malaria trend, P. falciparum, P. vivax, Ethiopia

\section{Background}

In Ethiopia, according to the ministry of health report, Plasmodium falciparum (P. falciparum) accounts for 55\% and Plasmodium vivax (P. vivax) for $45 \%$ of the cases. Reports also depicted that clinical malaria accounts for $10-40 \%$ of all out patient consultations and morbidity among children under 5 years in age being 10-20\% [1].

In Ethiopia, four major intervention strategies are still applied to combat malaria [2]. Moreover, awareness creation and community mobilization were used as a main strategy for the control and prevention purposes. Despite these activities, malaria remains a major killer disease in the study area. Previously, chloroquine was the most commonly used antimalarial drug. However; increased in resistance to $P$. falciparum resulted in a change for treatment. As

\footnotetext{
*Correspondence: yimermulat37@gmail.com

${ }^{1}$ Department of Medical Microbiology, Immunology and Parasitology, College of Medicine and Health Sciences, Bahir Dar University, Bahir Dar, Ethiopia

Full list of author information is available at the end of the article
}

a result, artemether/lumefantrine $\left(\right.$ Coartem $\left.{ }^{\circledR}\right)(\mathrm{AL})$ was adopted $[3,4]$. On the other hand, chloroquine is still the drug of choice for treatment of $P$. vivax even if, its resistance has not been yet known. Above all, self-treatment is common practice and this will result in development of drug resistance for the commonly prescribed drugs.

On top of these, different reports depicted confused results on decrement of malaria. Moreover, knowing the trends of malaria prevalence at each health institution is essential to design appropriate interventions. Furthermore, there is no information yet regarding the trends of malaria prevalence at health institution and particularly at Felegehiwot referral Hospital. Therefore, the present study addressed the above gaps.

\section{Methods \\ Study area}

A retrospective study was conducted to determine a 5 year trend analysis of malaria prevalence with in the catchment areas of Felegehiwot referral Hospital by reviewing malaria blood film malaria reports from 2010 
to 2014. Bahir Dar city is situated on the Southern shore of Lake Tana, the source of the Blue Nile in what was previously the Gojjam province and now the Amhara National Regional State. The city is located approximately $578 \mathrm{~km}$ Northwest of Addis Ababa, having an elevation of 1840 meters above sea level. Based on the 2007 Census conducted by the Central Statistical Agency of Ethiopia, it has a total population of 221,991, of whom 108,456 are males and 113,535 females [5]. Since, Felegehiwot Hospital is a referral Hospital, malaria cases might come from malaria stable and unstable transmission areas.

\section{Data collection}

5 year data on malaria cases were obtained from Felegehiwot referral Hospital. Laboratory technologists who examined blood films have 7-10 years of experience. To maintain the validity of this examination, a well-prepared and well-stained thin and thick blood films are used as the gold standard in confirming the presence of the malaria parasite as WHO protocol. The staining techniques and blood film examination for malaria parasite detection were conducted according to a standard operating procedure (SOP) stated on manual for the laboratory diagnosis of malaria [6] in each referral hospital throughout the country. Therefore, from July to August 2014, data on sex, age, type of Plasmodium species and season were collected on suspected malaria cases requested from 2010 to 2014 at Felegehiwot referral Hospital.

\section{Data analysis}

Data was analysed using Statistical Package for Social Sciences (PSS) version 20. Descriptive statistics such as line and bar graphs were used to show the trends of malaria in terms of season and year, respectively. Chisquare test was used to compare the trends of malaria, male and female and also between different age groups. Finally, $\mathrm{p}<0.05$ was considered as statistical significant.

\section{Results}

For the last 5 years (2010-2014), a total of 14,750 blood films were diagnosed for malaria at Felegehiwot referral Hospital. Of these, 7, 329 (49.7\%) were males and 7, 421
(50.3\%) were females with the median age of 23 . The overall prevalence of microscopy-confirmed malaria parasitaemia was 740 (5\%). Of these, P. falciparum accounted for 397 (2.7\%) and P. vivax for 331 (2.2\%). Plasmodium species infection had been observed more in males 406 (2.8\%) than females $334(2.3 \%)$. The difference was statistical significant $(\mathrm{p}<0.025)$ (Table 1$)$.

Trends of malaria by age group revealed that in $<5$ age groups, infection with $P$. falciparum was highest $58(14.6 \%)$ as compared to $P$. vivax 39 (12\%) and mixed infections $3(2.5 \%)$. In age groups from 5 to 9 and 10 to 15 , infection with $P$. vivax was dominant. In age groups from 16 to 20 and $>20$, there was a sharp increment in infection with both $P$. falciparum and $P$. vivax. Increments in mixed infections were observed from age groups 10-15 through $>20$ year. Statistical significant association was observed between age groups and infection with $P$. falciparum and $P$. vivax $(\mathrm{p}<0.02)$ (Fig. 1$)$.

A 5 year monthly trends of malaria showed that infection with $P$. falciparum was highest from September through November followed by February through May. More infection with $P$. vivax was observed from December to January followed by June through August. On the

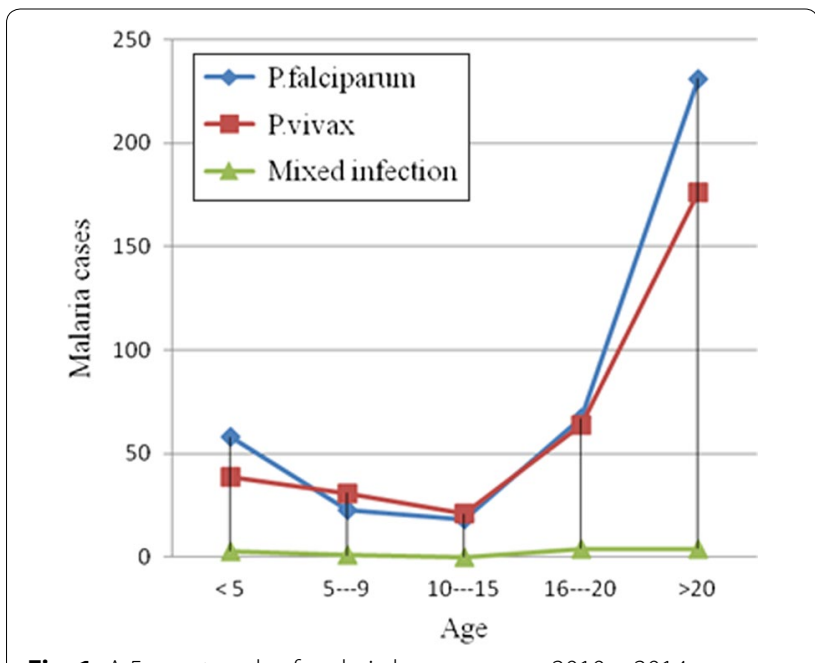

Fig. 1 A 5 year trends of malaria by age group, 2010-2014

Table 1 Prevalence of malaria by sex at Felegehiwot referral Hospital for the last 5 years (2010-2014)

\begin{tabular}{|c|c|c|c|c|c|c|}
\hline \multirow[t]{2}{*}{ Sex } & \multicolumn{6}{|l|}{ Plasmodium species } \\
\hline & P. falciparum no (\%) & P. vivax no (\%) & Mixed no (\%)* & Negative no (\%) & Total no (\%) & $p$ value \\
\hline Male & $216(2.9)$ & $185(2.5)$ & $5(0.07)$ & 7087 (95.5) & $7421(50.3)$ & $<0.025$ \\
\hline Female & $181(2.5)$ & $146(2)$ & $7(0.1)$ & $6923(94.5)$ & $7329(49.7)$ & \\
\hline Total & $397(2.7)$ & $331(2.2)$ & $12(0.08)$ & $14,010(95)$ & $14,750(100)$ & \\
\hline p value & 0.01 & & & & & \\
\hline
\end{tabular}

* Mixed infections malaria cases positive for both $P$. falciparum and $P$. vivax 


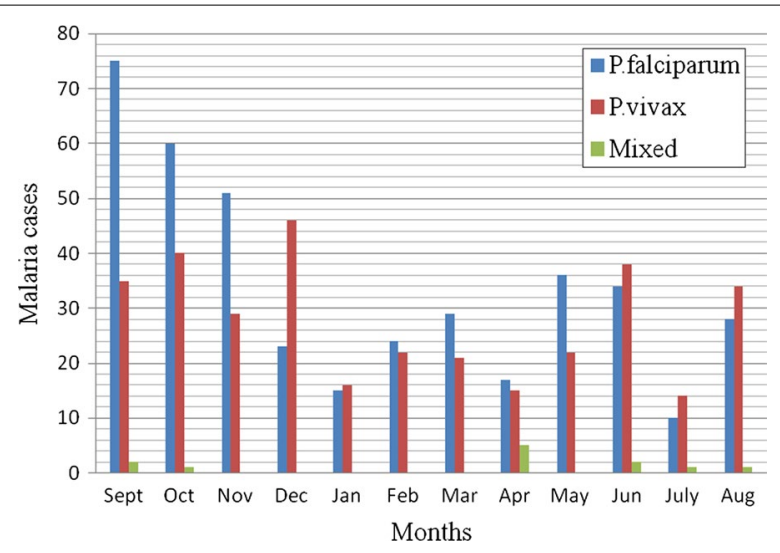

Fig. 2 Monthly trends of malaria for a 5 year period at Felegehiwot referral Hospital from 2010-2014

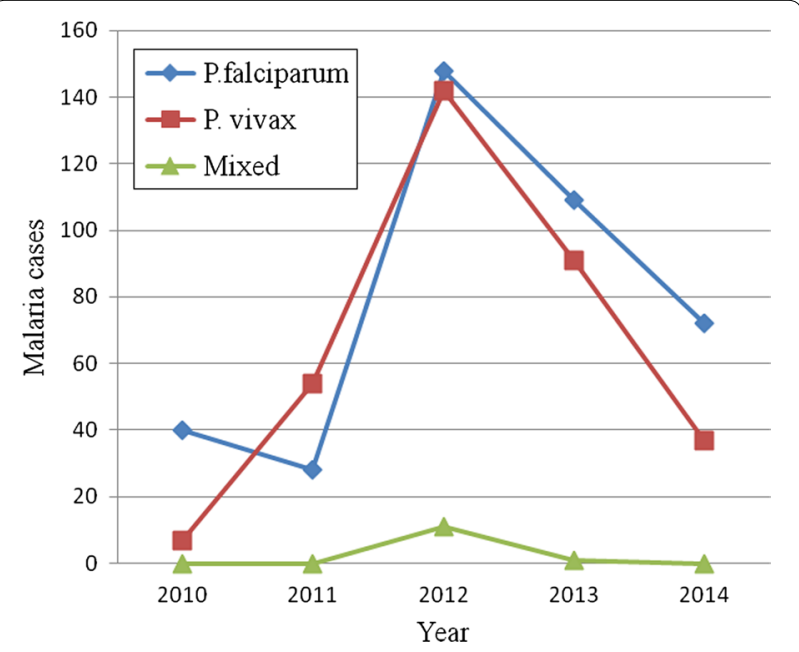

Fig. 3 Species trends of malaria parasites at Felegehiwot referral Hospital from 2010-2014

other hand, mixed infection was observed in September to October, in April and from June through August (Fig. 2).

Maximum cases with $P$. falciparum, $P$. vivax and mixed infections were observed in 2012 where as the least were observed in 2010. From 2011 to 2012, there was a sharp increment in $P$. vivax than $P$. falciparum infections. However, from 2012 through 2014, fast decrement in P. vivax than $P$. falciparum infections were observed. Finally, from 2011 to 2012, there was increment in mixed infections and from 2012 to 2013 there was decrement (Fig. 3).

\section{Discussion}

The present study revealed that microscopy- confirmed malaria in 5 year at Felegehiwot referral Hospital was 740 (5\%). This finding was smaller than a retrospective study done in Northwest Ethiopia [6] and Southwest Ethiopia
[7-9]. This difference might be due to study period and geographical difference. It was also smaller than the National Ministry of Health report [1]. This might be due to data on Ministry of Health is reports from via out the country while our report is from the study area. Moreover, there might be inter-personal variation on malaria microscopists and this might affect the result.

In this study, the overall prevalence of malaria in males 406 (2.8\%) was higher than females 334 (2.3\%). This result was in line with the study done in Southwest Ethiopia [8]. This might be due to the fact that agriculture is the main job and sleeping and staying outdoor is common during the night time. And hence, males are more exposed to Anopheles mosquito bites.

The prevalence of P. falciparum 397 (2.7\%) and P. vivax $331(2.2 \%)$ in the present study was lower than studies reported from other parts of the country $[8,10]$. This difference might be due to climatological differences and altitude variation.

In our study, the age groups, $>20$ years 411 (55.5\%) were highly affected followed by $16-20$ years old 135 (18.2\%) but from 10 to 15 years old 39 (5.2\%) were the least affected. This study coincides with a study done in Northwest [7], but different from a report in Southwest Ethiopia [9]. The possible reason might be due to responsibility of these age groups for caring of the family and hence, the probabilities of staying outdoor for a longer period.

Months and seasonality have a direct role in the transmission of malaria. In our study, the prevalence of $P$. falciparum throughout the year revealed that it seems stable transmission. The reason might be since Felegehiwot Hospital is a referral Hospital; new cases could be referred from malaria stable transmission areas. The highest prevalence was observed from September to December followed by May to June. This result was in agreement with the monthly trends of malaria transmission stated in ministry of health [11]. This monthly occurrence indicates the real malaria transmission.

According to this study, there were irregular occurrences of cases each year for the last 5 years. From 2011 to 2012, more $P$. vivax infection was observed compared to $P$. falciparum with the peak at 2012 which seems epidemic. This was in line with a report in Southwest Ethiopia [9]. This might be due to an overlooked feature of $P$. vivax $[11,12]$. The limitation of this study was that we used microscopy having lower sensitivity rather than polymerase chain reaction (PCR) to identify Plasmodium species in the study area.

\section{Conclusions}

The 5 year prevalence of malaria in our study area was lower than the National Ministry of Health. Therefore, more work will be done using PCR and RDT tests having more sensitivity. 


\section{Authors' contributions}

MY carried out drafting the proposal, involved in data collection and analysis up to final submission. TH, WM, WA and BA participated in proposal review, data analysis and final result review up to final submission. All authors read and approved the final manuscript.

\section{Authors' information}

MY is associate professor at College of Medicine and Health Sciences, Bahir Dar University in Medical Parasitology unit and head of Medical Parasitology unit. TH is assistant professor at College of Medicine and Health Sciences, Bahir Dar University in Medical Parasitology unit. WM is associate professor at College of Medicine and Health Sciences, Bahir Dar University in Medical Microbiology unit. WA is a Medical laboratory technologist at Felegehiwot referral hospital, Amhara National Regional State health Bureau. BA is an associate professor at College of Medicine and Health Sciences, Bahir Dar University in Medical Microbiology unit and department head of Microbiology, Immunology and Parasitology.

\section{Author details}

${ }^{1}$ Department of Medical Microbiology, Immunology and Parasitology, College of Medicine and Health Sciences, Bahir Dar University, Bahir Dar, Ethiopia.

${ }^{2}$ Amhara National Regional State Health Bureau, Felegehiwot referral Hospital, Bahir Dar, Ethiopia.

\section{Acknowledgements}

We would like to express great thanks to Felegehiwot referral hospital laboratory professionals and our colleagues: Mrs. Dagmawi Mengesha and Derjew Zewudie who assisted us for data collection along authors.

\section{Competing interests}

The authors declare that they have no competing interests.

\section{Availability of data and materials}

Since all data are already found in the manuscript and there is no additional files. The original data supporting this finding will be available at any time upon request.

\section{Consent for publication}

Consent to publish is not applicable for this manuscript because there is no individual data details.

\section{Ethics approval and consent to participate}

Ethical clearance was obtained from ethical and research review committee of Bahir Dar University, College of Medicine and Health Sciences. Permission letters were obtained from Amhara National Regional State Health Bureau and hospital director office prior to data collection.

\section{Publisher's Note}

Springer Nature remains neutral with regard to jurisdictional claims in published maps and institutional affiliations.

Received: 16 February 2016 Accepted: 19 June 2017

Published online: 04 July 2017

\section{References}

1. Federal Democratic Republic of Ethiopia Ministry of Health. Malaria: diagnosis and treatment guidelines for health workers in Ethiopia. Addis Ababa; 2012. pp 1-155.

2. Jima D, Medhin A. Malaria prevention and control in Ethiopia: progress and prospects. Fed Democr Repub Ethiop Minist Health Quart Health Bull. 2008;1:10-8.

3. World Health Organization. Assessment and monitoring of antimalarial drug efficacy for the treatment of uncomplicated falciparum Malaria. 2003. http://malaria.who.int/docs/ProtocolWHO.pdf.

4. EHNRI, MOH. Manual for the laboratory diagnosis of malaria. Addis Ababa; 2012. pp 1-126.

5. Federal Democratic Republic of Ethiopia Population Census Commission. Summary and statistical report of the 2007 population and housing census: population size by age and sex. Addis Ababa: Centreral Statistics Agency; 2008.

6. Alemu A, Muluye D, Mihret M, Adugna M, Gebeyaw M. Ten year trend analysis of malaria prevalence in Kola Diba, North Gondar, Northwest Ethiopia. Para Vect. 2012;5(173):1-6.

7. Sena LD, Deressa W, Ali M. Analysis of trend of malaria prevalence in south-west Ethiopia: a retrospective comparative study. Malaria J. 2014;13(188):1-9.

8. Asnakew KY, Sucharita G, Afework TH, Dereje OD, Hrishikesh PP. Spatial analysis of malaria incidence at the village level in areas with unstable transmission in Ethiopia. Int J Health Geogr. 2009;8(5):1-16.

9. Mula P, Fernández-Martínez A, de Lucio A, Ramos JM, Reyes F, González $V$, Benito A, Berzosa P. Detection of high levels of mutations involved in anti-malarial drug resistance in Plasmodium falciparum and Plasmodium vivax at a rural hospital in southern Ethiopia. Malaria J. 2011;10(214):1-7.

10. Alemu A, Fuehrer HP, Getnet G, Tessema B, Noedl H. Plasmodium ovale curtisi and Plasmodium ovale wallikeri in North-West Ethiopia. Malaria J. 2013;12(346):1-7.

11. Hay SI, Guerra CA, Tatem AJ. The global distribution and population at risk of malaria: past, present, and future. Lancet Infect Dis. 2004:4:327-36.

12. Baird JK. Neglect of Plasmodium vivax malaria. Trends Parasitol. 2007:23:533-9.

\section{Submit your next manuscript to BioMed Central and we will help you at every step:}

- We accept pre-submission inquiries

- Our selector tool helps you to find the most relevant journal

- We provide round the clock customer support

- Convenient online submission

- Thorough peer review

- Inclusion in PubMed and all major indexing services

- Maximum visibility for your research

Submit your manuscript at www.biomedcentral.com/submit
Ciomed Central 